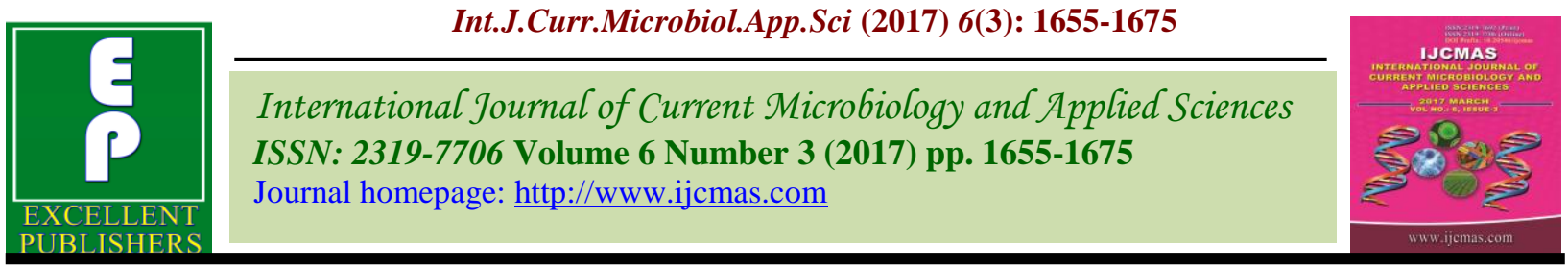

Original Research Article

https://doi.org/10.20546/ijcmas.2017.603.192

\title{
Identification and Characterization of Bile Salt Hydrolyzing Lactobacillus Isolates
}

\section{Pradip Kumar Sharma ${ }^{1 *}$, Pradeep Kumar Sharma ${ }^{1}$ and Naresh Kumar ${ }^{2}$, Suman $^{2}$ and Niti Dhingra ${ }^{3}$}

\author{
${ }^{1}$ Department of Microbiology, Ch. Charan Singh University Meerut, India \\ ${ }^{2}$ Dairy Microbiology Division, National Dairy Research Institute, Karnal, India \\ ${ }^{3}$ Department of Biotechnology, DAV College, Sec, 10, Chandigarh, India \\ *Corresponding author
}

\section{A B S T R A C T}

\section{Keywords}

Probiotic,

Lactobacillus, BSH

activity, Molecular

identification.

Article Info

Accepted:

24 February 2017

Available Online:

10 March 2017
Thirty nine, out of 46 acid tolerant Lactobacillus spp., isolated from traditional dairy products, fermented foods and human fecal samples, were preliminary identified at genus level as Lactobacillus and evaluated for probiotic properties which included bile tolerance, simulated gastrointestinal juice, cell surface hydrophobicity, cell auto-aggregation, coaggregation and bile salts hydrolase activity. Thirty three isolates could resist well at $1.5 \%$ bile while 26 isolates showed tolerance towards simulated gastric juice $(\mathrm{pH} 2.0,3 \mathrm{~h}$ of incubation) and simulated pancreatic juice ( $\mathrm{pH} 8.0$ ). Only 9 isolates could exhibited $>50 \%$ cell surface hydrophobicity wherein LBF89, LBF91 and LBF90 exhibited highest cell surface hydrophobicitywith n-hexadecane i.e. $73.47 \pm 2.15 \%, 72.25 \pm 1.69 \%$ and $71.65 \pm 1.90 \%$ respectively. LBF89 showed highest cell auto-aggregation $(50.67 \pm 1.08 \%)$ while least auto-aggregation was demonstrated by LBF 11. LBF 20 exhibited highest mean co-aggregation $50.17 \%$ with E. coli, L. monocytogenes, S. abony and S. aureus. S. abony was the highly encountered pathogen with an average value of $31.14 \%$ ofco-aggregation. Highest bile salt hydrolase activity was observed in LBF 89 and LBF 91with sodium taurodeoxycholate. Bile salt hydrolase activity was quantitatively determined wherein the highest activity i.e.7.21 \pm 0.10 , was observed in LBF 89 isolate followed by LBF 91 with $6.56 \pm 0.10$ as total enzyme activity. Both the isolates were identified using PCR as Lactobacillus plantarum but distantly placed in the phylogenetic tree. Among the selected isolates, LBF 89 and LBF 91 showed the best probiotic potential with high tolerance to bile, simulated gastrointestinal juice and exhibited high bile salt hydrolase activity. Both the isolates possess application potential for functional foods and health-associated products.

\section{Introduction}

In recent years, different investigations support the importance of probiotics as a part of healthy diet for humans and animals and as a way to provide a natural, safe and effective barrier against microbial infections (Angmo et al., 2016). World Health Organization
(WHO) has laid down the definition of probiotics as "live microbial food supplements which, when administered in adequate amounts confer health benefit on the host" (FAO/WHO, 2001). Among the usually used microorganisms, lactic acid bacteria 
(LAB) are regarded as a major group of probiotic bacteria (Collins and Gibson, 1999). LAB is non-pathogenic, technologically suitable for industrial processes, acid \& tolerant and produce antimicrobial substances (Mojgani et al., 2015).

LAB are classified as generally recognized as safe (GRAS) microorganisms because of their long and safe use as starter cultures in fermented food products. Most of the probiotic organisms belong to the genera Lactobacillus and Bifido bacterium (Prasad et al., 1998), however, species belonging to the genera Lactococcus, Enterococcus and Saccharomyces (Salminen and von Wright, 1998; Sanders and in't Veld, 1999) are also considered as probiotic microorganisms. As per the recommendations of Joint FAO/WHO working group, two currently most widely used in vitro tests for selection of probiotics are resistance to gastric acidity and bile salts, as evident by survival and growth studies, (Vijaya et al., 2015). Other functional properties for characterization of probiotics are production of antimicrobial compounds and cholesterol assimilation (Park et al., 2007; Xie et al., 2015).

The mechanism through which probiotics may antagonize pathogens involves production of antimicrobial compounds such as lactic acid, acetic acid, hydrogen peroxide and bacteriocins. Other properties of probiotic organisms include, reduced deportment of pathogenic microorganism, lowered risk factors for coronary artery disease and a dose dependent reduction in the symptoms of irritable bowel syndrome (Vries et al., 2006). Several probiotics bacteria are found to produce bile salt hydrolase $(\mathrm{BSH})$ that helps to reduce serum cholesterol (Miremadi et al., 2014) and hence BSH activity is also considered as an additional criterion for the selection of probiotics.

\section{Materials and Methods}

Forty six acid tolerant, gram positive, catalase negative strains isolated from traditional dairy products, fermented foods and human fecal samples were taken under this study. These isolates were selected as acid tolerant after extensive study for their survival towards different $\mathrm{pH}$ i.e. 2.0, 3.0 and 6.5 (data not shown). The selected isolates were grown in de Man, Rogosa, and Sharpe (MRS) broth at $37{ }^{\circ} \mathrm{C}$ for $16-18 \mathrm{~h}$ and sub-cultured twice prior to conducting the experiments. E. coli, L. monocytogenes, S. abony and S. aureus used in co-aggregation study, were obtained from ATCC.

\section{Molecular characterization at genus level}

Genomic DNA was isolated using GeNei pure Bacterial DNA purification kit as per manufacturer's instructions. Genus specific PCR primers LbLMA1-rev 5'-CTC AAA ACT AAA CAA AGT TTC-3' (specific primer) and R16-1 -5'-CTT GTA CAC ACC GCC CGT CA-3, corresponding to the flanking terminal sequence of the $16 \mathrm{~S}$ rRNA gene giving rise to $250 \mathrm{bp}$ PCR product (Dubernet et al., 2002) were used for preliminary identification of the isolates as lactobacilli using PCR (Bile tolerance).

Bile tolerance was tested according to the method described by Gilliland et al., (1984).Overnight activated culture of lactobacilli isolates were harvested by centrifugation $\left(7000 \mathrm{x} \mathrm{g}\right.$ at $4^{\circ} \mathrm{C}$ for $\left.10 \mathrm{~min}\right)$ and re-suspended in equal volume of MRS broth supplemented with $0.5 \%, 1.0 \%$ and 1.5 $\%$ of ox bile (Hi-media) and incubated at 37 ${ }^{\circ} \mathrm{C}$. Broth without ox bile served as control. The survival $(\%)$ of the isolates at different time interval was calculated as follows: Survival $\%=($ Number of viable cells survived/Number of initial viable cells 
inoculated) x100 (Tambekar and Bhutada, 2010).

\section{Survival under simulated juice}

To test the viability in presence of pepsin, simulated gastric juice was prepared by suspending $3.0 \mathrm{mg} / \mathrm{mL}$ pepsin in sterile saline solution $(0.85 \% \mathrm{NaCl}, \mathrm{w} / \mathrm{v})$ and $\mathrm{pH}$ was adjusted to 2.5. Simultaneously, for assessing survival ability in presence of pancreatin, simulated intestinal fluid was prepared by dissolving bile salt $\mathrm{Na}$ Taurodeoxycholate $(0.3 \%)$ and pancreatin $(1.0 \mathrm{mg} / \mathrm{mL})$ in sterile saline solution $(0.85 \%$ $\mathrm{NaCl}$, w/v) adjusted to $\mathrm{pH} 8.0$. The whole preparation was sterilized by passing through $0.22 \mu \mathrm{m}$ syringe filter (Kos, et al., 2000). Isolates were grown in MRS broth for 16-18 hours and cells were harvested using refrigerated centrifuge $\left(7000 \mathrm{x} \mathrm{g}\right.$ at $4^{\circ} \mathrm{C}$ for 10 min). Pellet was washed using potassium phosphate buffer and re-suspended in (10 $\mathrm{mM}, \mathrm{pH}$ 6.8) in same buffer to 1.0 OD using plate reader (Perkin Elmer).

One $\mathrm{mL}$ of each fluids were mixed with 200 $\mu \mathrm{L}$ of bacterial cell suspension and incubated at $37^{\circ} \mathrm{C}$. Afterwards, aliquots were taken at different time interval viz., $0 \mathrm{~min}, 90 \mathrm{~min}$ and $180 \mathrm{~min}$ and plated for total viable count using MRS Agar media. Survival (\%) of the isolates was calculated as follows: Survival $\%=$ (Number of viable cells survived / Number of initial viable cells inoculated) $\mathrm{x} 100$.

\section{Cell surface hydrophobicity}

Cell surface hydrophobicity (CSH)was determined as per the method described by Rosenberg et al., (1980) using n-hexadecane. For CSH, overnight grown cells in MRS broth were harvested by centrifugation at $(7000 \mathrm{x} \mathrm{g}$ at $4^{\circ} \mathrm{C}$ for $10 \mathrm{~min}$ ) and washed with potassium phosphate buffer (PB) $10 \mathrm{mM}, \mathrm{pH}$ 7.0. The pellet was re-suspended in the same buffer and adjusted to final OD of 0.7 at $595 \mathrm{~nm}$ absorbance using Perkin Elmer Victor X3 plate reader. Lactobacilli suspension $(3.0 \mathrm{~mL})$ and $\mathrm{n}$-hexadecane $(1.0 \mathrm{~mL})$ were taken in a tube and mixed by vortexing. The preparation was incubated at $37^{\circ} \mathrm{C}$ for $10 \mathrm{~min}$ for temperature equilibration.

The mixture was again vortex for a while and incubated at $37^{\circ} \mathrm{C}$ for 1.0 hour for phase separation. The hydrocarbon layer was allowed to rise completely. Aqueous phase was removed carefully and checked for absorbance using spectrophotometer $(595 \mathrm{~nm})$ after $10 \mathrm{~min}$ (initial OD) and $1.0 \mathrm{~h}$ (final OD). Cell surface hydrophobicity (\%) was calculated as follows: Cell surface hydrophobicity $(\%)=[(\mathrm{Abs}$ initial-Abs final)/Abs final)] $\times 100$.

\section{Cell auto-aggregation}

Auto-aggregation assay was performed according to Del Re et al (2000). Lactobacillus isolates were grown for $18 \mathrm{~h}$ at $37^{\circ} \mathrm{C}$ using MRS broth. Overnight grown cells were harvested from the broth by centrifugation at $\left(7000 \mathrm{x} \mathrm{g}\right.$ at $4^{\circ} \mathrm{C}$ for $\left.10 \mathrm{~min}\right)$ phosphate buffer (PB) $10 \mathrm{mM}, \mathrm{pH} 7.0$ and resuspended in the same buffer to an absorbance of 0.5 at $595 \mathrm{~nm}$ (Abs initial) to give viable counts of approximately $10^{8} \mathrm{CFU}$ $/ \mathrm{mL}$. The suspension was centrifuge and the pellet were re-suspended in equal volume of sterilized MRS broth.

The suspension were allowed to stand at $37^{\circ} \mathrm{C}$ for $5 \mathrm{~h}$. Afterwards, $1.0 \mathrm{~mL}$ of the upper suspension were taken to measure the absorbance (Abs final) by using sterile broth as reference. The percent difference between the initial and final absorbance was taken as an index of auto-aggregation using as following formula: Auto-aggregation \% = [(Absorbance initial-Absorbance final) /Absorbance initial] x 100 . 


\section{Co-aggregation}

Co-aggregation ability of each isolate was determined by the method described by Del Re et al., (2000). Each isolate was inoculated into MRS broth and incubated at $37^{\circ} \mathrm{C}$ for 18 hours. At the same time, the bacterial indicators which included $E$. coli, $L$. monocytogenes, S. abony and S. aureus were inoculated in $\mathrm{BHI}$ broth and incubated at $35^{\circ} \mathrm{C}$ for 16-18 h. Overnight grown cells of Lactobacillus isolates were harvested by centrifugation at $\left(7000 \mathrm{x} \mathrm{g}\right.$ at $4^{\circ} \mathrm{C}$ for $\left.10 \mathrm{~min}\right)$ and washed with potassium phosphate buffer (PB) $10 \mathrm{mM}, \mathrm{pH}$ 6.8. The pellet was resuspended in the same buffer so as to obtain $1.0 \mathrm{OD}(595 \mathrm{~nm})$. Indicator organisms were also spin and washed using P.B ( $\mathrm{pH}$ 6.8). The pellet was suspended in the same buffer so as to obtain 0.6 OD $(595 \mathrm{~nm})$. Suspension of Lactobacillus bacteria and pathogens were taken in $1: 1$ ratio and incubated at $35^{\circ} \mathrm{C}$ for 4 h. Suspension of individual isolate and pathogens were taken as respective controls. Absorbance of the suspension was monitored at $595 \mathrm{~nm}$ over a period of 4 hours. Coaggregation \% was calculated according to Handley's equation (Handley et al., 1987).

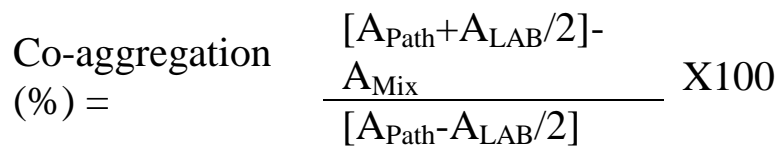

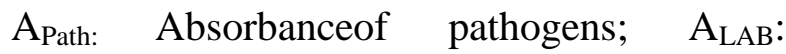
Absorbance of Lactobacillus isolates; $\mathrm{A}_{\text {Mix }}$ : Absorbance of mixture containing pathogens and Lactobacillus isolates.

\section{Bile salt hydrolase activity}

\section{Direct plate assay}

The qualitative Bile salt hydrolase (BSH) activity of the isolates was assessed by direct plate assay (Schillinger et al., 2005). Lactobacillus isolates were streaked on MRS agar plates supplemented with $0.5 \%(\mathrm{w} / \mathrm{v})$ filter sterilized bile salts (sodium taurodeoxycholate hydrate; sodium taurocholate; sodium deoxycholate; deoxycholic acid and cholic acid; Sigma, USA) along with $0.37 \mathrm{~g} / \mathrm{L}$ of $\mathrm{CaCl}_{2}$. The plates were incubated anaerobically in an anaerobic gas pack jar at $37^{\circ} \mathrm{C}$ for $72 \mathrm{~h}$. MRS agar plates without bile salt supplementation were used as control. The presence of precipitated bile acid around colonies (opaque halo) or the formation of opaque granular white colonies with a silvery shine was considered as a positive reaction

\section{Quantitative assay for bile salt hydrolase activity}

The bile salt hydrolase (BSH) assay was also performed by measuring amino acids released from hydrolysis of conjugated bile salts by Lactobacillus isolates as described by Liong and Shah, (2005). Exponentially growin Lactobacilli cells were harvested at $(7000 \mathrm{x} \mathrm{g}$ at $4^{\circ} \mathrm{C}$ for $10 \mathrm{~min}$ ), washed twice with $0.1 \mathrm{M}$ sodium phosphate buffer $(\mathrm{pH}$ 6.8) and resuspended in same buffer to $1.0 \mathrm{OD}$ at 600 $\mathrm{nm}$. Five $\mathrm{mL}$ of bacterial suspension were sonicated for 3.0 min with constant cooling using ice followed by centrifugation at 10,000 $\mathrm{rpm}$ at $4^{\circ} \mathrm{C}$ for $10 \mathrm{~min}$. The reaction mixture contained $100 \mu \mathrm{L}$ cell suspension, $100 \mu \mathrm{L}$ of $10 \mathrm{mM}$ conjugated bile salt (sodium glycocholate) and $1.8 \mathrm{~mL}$ of $0.1 \mathrm{M}$ sodium phosphate buffer ( $\mathrm{pH}$ 6). Reaction mixtures were incubated at $37^{\circ} \mathrm{C}$ for $30 \mathrm{~min}$. Equal volume of reaction mixture and $15 \%$ trichloroacetic acid (TCA,w/v, $200 \mu \mathrm{L}$ each) were taken and centrifuge at $12,000 \mathrm{rpm}$ for $15 \mathrm{~min}$ at $4^{\circ} \mathrm{C}$. The amount of amino acids present in the supernatant was measured. An aliquot of $0.2 \mathrm{~mL}$ of supernatant obtained after centrifugation was added to $1.0 \mathrm{~mL}$ of distilled water and $1.0 \mathrm{~mL}$ of ninhydrin reagent $(0.5 \mathrm{~mL}$ of $1.0 \%$ ninhydrin in $0.5 \mathrm{M}$ citrate buffer, $\mathrm{pH} 5 \cdot 5,1.2 \mathrm{~mL}$ of $30 \%$ 
glycerol and $0.2 \mathrm{~mL}$ of $0.5 \mathrm{M}$ citrate buffer, $\mathrm{pH}$ 5.5). The preparation was vortex vigorously and kept in dry block heater at 95 $100{ }^{\circ} \mathrm{C}$ for $14 \mathrm{~min}$. After cooling, absorbance was determined at $570 \mathrm{~nm}$ using glycine as standards. One unit of BSH activity $(\mathrm{U} / \mathrm{mL})$ was defined as the amount of enzyme that liberated $1 \mathrm{nmol}$ of amino acid substrate per min per absorbance at $570 \mathrm{~nm}$.

Molecular identification of selected Lactobacillus isolates at species level

\section{Polymerase chain reaction}

Amplification of selected Lactobacillus isolates was executed using universal prokaryotic primers for the 16S rRNA gene pA (5'-AGAGTTTGATCCTGGCTCAG; nucleotide 8 to 27 of the 16S rRNA gene of $E$. coli) and $\mathrm{pH}$ (5'AAGGAGGTGATCCAGCCGCA;

nucleotide 1541 to 1522 of the 16S rRNA gene of E. coli; (Rodas et al., 2003) yielding a PCR product of $\sim 1500 \mathrm{bp}$.

\section{Gel electrophoresis of PCR products and sequencing}

A 100-1500 bp ladder (Fermentas,) was used as a molecular mass marker. Bands appearing at $1500 \mathrm{bp}$ were considered for sequencing followed by BLAST analsyis.PCR products were sent to M/s. Invitrogen Bioservices India Pvt. Ltd. Udhog Vihar, Gurgaon, India and were directly sequenced using the forward and reverse primers. The sequences were further used to identify the isolate at species level by using basic local alignment sequence tool (BLAST) for similarity search at NCBI website using GenBank data (Altschul et al., 1997).

\section{Statistical analysis}

Data were statistically analyzed with GraphPad Prism 5.1 software. One-way analysis of variance was used to study significant difference between means, with significance level at $\mathrm{P}=0.05$.

\section{Results and Discussion}

\section{Preliminary identification of isolates by genus specific PCR}

Molecular methods are more reliable and accurate than that of biochemical ones. Fig 1 shows 250 bp PCR product obtained with 39 isolates out of 46 acid tolerant Lactobacillus isolates. Reference Lactobacillus culture was also used as a positive control. However, 8 isolates were failed to amplify using the above procedure showing that they may not be of genus Lactobacillus.

\section{Tolerance against bile}

Bile resistance is one of the important criteria for selection of probiotic (Lee and Salminen, 1995; Dunne et al., 2001). Resistance to bile helps probiotic bacteria to reach the small intestine and colon and subsidize in balancing the intestinal microflora (Tambekar and Bhutada, 2010). After 3 hour of incubation, At $0.5 \%$ bile, 5 isolates LBF 08, LBF 20, LBF 11, LBF 01 and LBF197 showed $70 \%$ or above survival which is very close to LGG $(81.22 \pm 0.75 \%)$. Moreover, 20 isolates showed more than $60 \%$ survival ranging $61.2 \%-69.4 \% ; 8$ isolates with more than $50 \%$ survival $(52.5 \%-59.53 \%)$ while remaining 06 isolates exhibited survival between $22.0-36.0$ $\%$.

Whereas, 9 isolates showed $60 \%$ or above survival $(61.0 \%-76.1 \%)$ at $1.0 \%$ bile concentration which is comparable to LGG $(76.10 \pm 0.62 \%)$ after 3 hours of incubation. In addition to this, 22 isolates showed more than $50 \%$ survival $(51.46 \%-59.95 \%)$ while 2 isolates with more than $40 \%$ survival. Only, 06 isolates exhibited 18.0-29.0 \% survival at $1.0 \%$ bile concentration. 
At highest concentration of bile i.e. $1.5 \%$, only 5 isolates showed 41.19-42.36\% survival along with LGG $(46.19 \pm 0.68 \%)$ after 3 hours of incubation. Moreover, 25 isolates showed $31.37 \%-39.79 \%$ and 3 isolates with $26.21 \%-28.47 \%$ survival. Apart from this, 6 isolates exhibited less than $10 \%$ survival (Table 1). The isolates which tolerated $1.5 \%$ of bile concentration after 3 hours exposure with $10 \%$ or above survival were considered as bile tolerant and selected for further investigation (Pennacchia, 2004). A total of 33 isolates were selected out of 39 isolates for further characterization.

\section{Survival under simulated gastric and pancreatic juice}

During transit from oral to colon, probiotic bacteria are supposed to survive through stomach followed by intestine and exert their health promoting effects as metabolically viable active cells after reaching in the colon (Malek et al., 2010).All 33 isolates were able to survive under simulated gastric juice containing pepsin at $\mathrm{pH} 2.0$ up to $3 \mathrm{~h}$ of incubation with variable survival rates. Out of 33 isolates, only 2 isolates (LBF 92 and LBF 198) registered survival of $72.53 \pm 0.50 \%$ and $73.16 \pm 0.54 \%$, respectively. In addition to this, 13 Lactobacillus isolates showed more than $60.0 \%$ survival but less than $70 \%$ survival ranging from $60.46 \pm 0.41 \%$ to $69.71 \pm 0.45 \%$ with variable degree of $\log$ reduction. LGG showed $68.12 \pm 0.84 \%$ survival under these conditions which is comparable to 13 different isolates. Apart from this, 12 Lactobacillus isolates exhibited survival between 50-60\% ranging from $52.17 \pm 0.43$ to $59.62 \pm 0.49 \%$. However, 6 isolates showed less than $20 \%$ survival ranging from $6.71 \pm 0.06 \%$ to $18.97 \pm 0.09 \%$ (Table 2).

Under simulated pancreatic juice ( $\mathrm{pH} \mathrm{8.0),} \mathrm{all}$ 33 isolates were able to survived by $3 \mathrm{~h}$ of incubation with variable degree of $\log$ reduction. Out of 33 isolates, 2 isolates (LBF 01 and LBF 20) exhibited $23.30 \pm 0.26 \%$ and $21.26 \pm 0.18 \%$ survival which is comparable with reference culture LGG $(22.19 \pm 0.27 \%)$. Moreover, 19 Lactobacillus isolates were able to withstand the pancreatic juice with survival ranging from $10.26 \%$ to $19.83 \%$ survival. In addition to this, 7Lactobacillus isolates $(21.21 \%)$ revealed less than $10 \%$ ranging from 1.59 to $9.35 \%$ while 5 Lactobacillus isolates showed below $1.0 \%$ ranging from $0.30 \%$ to $0.91 \%$ (Table 2). These 5 isolates also revealed less survival for simulated gastric juice. Overall, only 27 isolates were selected after scrutiny of tolerance towards bile and good survival under simulated gastric and pancreatic juice.

\section{Cell surface hydrophobicity}

The colonization in intestinal wall is considered as one of the prominent criteria for selection of probiotics. CSH was assessed by measuring the adhesion ability of the isolates to the intestinal epithelium (NiguezPalomares et al., 2008; Tuomola et al., 2001).

Significant differences in hydrophobicity were observed within Lactobacillus isolates. Out of 27 isolates, 3 isolates LBF89, LBF91 and LBF90 exhibited highest value of $\mathrm{CSH}$ i.e. $73.47 \pm 2.15 \%, 72.25 \pm 1.69 \%$ and $71.65 \pm 1.90 \%$, respectively, which is very close to the reference strain LGG $(74.10 \pm 2.24 \%)$ with n-hexadecane. In addition to this, 4 Lactobacillus isolates, designated as LBF 05, LBF 01, LBF 08 and LBF 11 showed $65.45 \pm 2.15 \%, 64.72 \pm 2.93$ $\%, 63.41 \pm 2.05 \%$ and $62.87 \pm 2.09 \% \mathrm{CSH}$, respectively. LBF 20 and LBF 13A also confirmed respectable level $(58.87 \pm 2.06 \%$ and $56.38 \pm 2.16 \%$, respectively) of $\mathrm{CSH}$. However, rest 17 isolates exhibited CSH in the range of $1.73 \%$ to $21.69 \%$ (Table 3 ). 


\section{Cell auto aggregation}

The auto-aggregation ability of 9 Lactobacillus isolates along with LGG was studied. LGG showed highest cell autoaggregation with $50.68 \pm 1.08 \%$ while least auto-aggregation was demonstrated by LBF 11 with $40.50 \pm 0.89 \%$. Other isolates also presented comparable value of cell autoaggregation to LGG. LBF89 showed $50.67 \pm 1.08 \%$ followed by LBF90 $(48.99 \pm 0.97 \%)$, LBF 13 A $(48.01 \pm 0.89 \%)$, LBF91 (46.52 $\pm 0.90 \%)$, LBF $01(44.66 \pm 1.07$ $\%), \quad$ LBF $20 \quad(43.38 \pm 0.96 \%), \quad$ LBF 08 $(41.03 \pm 0.74 \%)$ and LBF $05(40.51 \pm 0.95 \%)$. (Table 4). The isolates complies the criteria as recommended by Del Re et al., (2000) wherein $35-40 \%$ of auto-aggregation has been recommended for an isolate to be a good probiotic.

\section{Co-aggregation}

The co-aggregation ability of 09 Lactobacillus isolates with $E$. coli, $L$. monocytogenes, $S$. abony and $S$. aureuswas studied. LBF 08, LBF 89, LBF 90 and LBF 91 exhibited very virtuous co-aggregation with the pathogens taken into consideration. LBF 20 exhibited highest mean coaggregation $50.17 \%$ for all pathogens followed by LBF 89(33.56 \%), LBF 90 and 91(30.72\%) while remaining isolates showed below $20 \%$ ranging 10/96 \%-17.50\%. S. abony was the highly encountered pathogen for co-aggregation with an average value of $31.14 \%$ followed by $E$. coli $(27.56 \%), S$. aureus $(23.60 \%)$ and L. monocytogenes $(21.27 \%)$ (Table 4).

\section{Bile salt hydrolase activity}

BSH activity of Lactobacillus isolates was assessed qualitatively using MRS agar supplemented with different bile salts. All the 09 isolates along with L. rhamnosus GG, showed differed results. Highest bile salt hydrolase activity was observed against sodium taurodeoxycholate as evident from intensity of precipitated opalescent zones, relatively higher in LBF 89 and LBF 91 (Table 5). The highest BSH enzyme activity was observed in LBF 89 isolate i.e. 7.21 \pm 0.10 as total enzyme activity comparatively higher then LGG (6.17 \pm 0.07$)$. Other isolates also exhibited significant BSH activity i.e. LBF 91 (6.56 \pm 0.10$)$, LBF 90 (5.55 \pm 0.23$)$, LBF 08

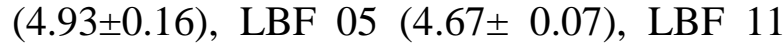
(3.27 \pm 0.17$),$ LBF 13 A $(2.16 \pm 0.16)$ and LBF $01(4.89 \pm 0.08)$ (Table 5). The results indicated possibility of presence of $b s h$ gene enabling the strain to hydrolyse bile salts and thus high efficient to remove bile salts from the body through fecal excretion.

\section{Molecular identification}

Fig 2 illustrates 1500 bp PCR product obtained with 9 isolates along with LGG. Based on 16S r-DNA sequencing data, LBF $01,13 \mathrm{~A}$ and LBF 90 were identified as $L$. fermentum, 95.0\%, 99.0\% and $100.0 \%$ homology, respectively, LBF 05 as $L$. helveticus (99.0\%), LBF 08 as L. acidophilus (99.0\%), LBF 11 as L. casei (99.0\%), LBF 20 as $L$. reuteri $(100.0 \%)$ homology, $\mathrm{LBF} 89$ as L.paraplantarum (99.0\%), and LBF 91 as $L$. plantarum $(99.0 \%)$ homology. The sequences were submitted to gene bank and accession numbers have been delineated in table 6 . The sequences were aligned using MEGA 6.0 software (Tamura et al., 2013) and phylogenetic tree was generated through accessing the relevant nucleotide sequences from NCBI nucleotide database (Fig 3).

Acid tolerant lactobacilli isolates were identified at genus level using Genus specific PCR primers LbLMA1 (specific primer) and R16-1. 
Table.1 Survival (\%) of Lactobacillus isolates after 3 hours of incubation at different bile concentration

\begin{tabular}{|c|c|c|c|c|c|c|}
\hline S. No. & Isolate No. & $0.5 \%$ Bile & \multicolumn{2}{|c|}{$1.0 \%$ Bile } & \multicolumn{2}{|c|}{$1.5 \%$ Bile } \\
\hline & LGG & $81.22 \pm 0.75$ & $76.10 \pm$ & 0.62 & $46.19 \pm$ & 0.68 \\
\hline 1. & LBF 01 & $73.42 \pm 0.78$ & $65.10 \pm$ & 0.76 & $41.51 \pm$ & 0.69 \\
\hline 2. & LBF 05 & $68.32 \pm 0.84$ & $62.10 \pm$ & 0.85 & $41.37 \pm$ & 0.63 \\
\hline 3. & LBF 06 & $65.42 \pm 0.89$ & $61.00 \pm$ & 0.69 & $38.27 \pm$ & 0.62 \\
\hline 4. & LBF 08 & $78.58 \pm 0.72$ & $68.61 \pm$ & 0.73 & $35.18 \pm$ & 0.76 \\
\hline 5. & LBF 11 & $73.68 \pm 0.71$ & $62.46 \pm$ & 0.69 & $32.13 \pm$ & 0.85 \\
\hline 6. & LBF 13 A & $68.98 \pm 0.78$ & $63.66 \pm$ & 0.73 & $39.18 \pm$ & 0.69 \\
\hline 7. & LBF 13 B & $55.86 \pm 0.72$ & $51.46 \pm$ & 0.62 & $32.21 \pm$ & 0.73 \\
\hline 8. & LBF 18 & $56.16 \pm 0.73$ & $52.11 \pm$ & 0.95 & $26.25 \pm$ & 0.69 \\
\hline 9. & LBF 19 & $58.26 \pm 0.93$ & $54.61 \pm$ & 0.68 & $28.47 \pm$ & 0.73 \\
\hline 10. & LBF 20 & $74.47 \pm 0.71$ & $68.45 \pm$ & 0.69 & $36.74 \pm$ & 0.63 \\
\hline 11. & LBF 23 & $52.50 \pm 0.65$ & $43.41 \pm$ & 0.63 & $39.79 \pm$ & 0.68 \\
\hline 12. & LBF 24 & $62.20 \pm 0.85$ & $56.33 \pm$ & 0.62 & $42.36 \pm$ & 0.62 \\
\hline 13. & LBF 26 & $63.56 \pm 0.78$ & $59.41 \pm$ & 0.76 & $33.79 \pm$ & 0.69 \\
\hline 14. & LBF30 & $35.06 \pm 0.62$ & $23.52 \pm$ & 0.65 & $2.50 \pm$ & 0.34 \\
\hline 15. & LBF34 & $26.26 \pm 0.95$ & $21.36 \pm$ & 0.67 & $4.72 \pm$ & 0.37 \\
\hline 16. & LBF35 & $61.20 \pm 1.02$ & $56.85 \pm$ & 0.86 & $33.42 \pm$ & 0.77 \\
\hline 17. & LBF48 & $32.50 \pm 1.15$ & $28.63 \pm$ & 0.69 & $3.92 \pm$ & 0.55 \\
\hline 18. & LBF49 & $35.60 \pm 0.79$ & $28.99 \pm$ & 0.64 & $4.56 \pm$ & 0.59 \\
\hline 19. & LBF50 & $27.86 \pm 0.87$ & $23.89 \pm$ & 0.83 & $3.65 \pm$ & 0.31 \\
\hline 20. & LBF52 & $22.46 \pm 0.82$ & $18.74 \pm$ & 0.54 & $3.51 \pm$ & 0.27 \\
\hline 21. & LBF53 & $62.46 \pm 0.84$ & $56.55 \pm$ & 0.72 & $26.21 \pm$ & 0.77 \\
\hline 22. & LBF59 & $65.29 \pm 0.89$ & $53.58 \pm$ & 0.86 & $33.22 \pm$ & 0.77 \\
\hline 23. & LBF81 & $64.79 \pm 0.91$ & $62.43 \pm$ & 0.89 & $41.28 \pm$ & 0.74 \\
\hline 24. & LBF82 & $63.30 \pm 0.79$ & $53.22 \pm$ & 0.84 & $33.40 \pm$ & 0.80 \\
\hline 25. & LBF83 & $65.50 \pm 0.86$ & $52.41 \pm$ & 0.73 & $32.39 \pm$ & 0.86 \\
\hline 26. & LBF84 & $56.07 \pm 0.73$ & $53.19 \pm$ & 0.69 & $33.24 \pm$ & 0.86 \\
\hline 27. & LBF85 & $59.53 \pm 0.79$ & $49.64 \pm$ & 0.73 & $35.22 \pm$ & 0.89 \\
\hline 28. & LBF87 & $58.43 \pm 0.85$ & $53.54 \pm$ & 0.63 & $35.58 \pm$ & 0.84 \\
\hline 29. & LBF89 & $56.71 \pm 0.84$ & $52.75 \pm$ & 0.68 & $35.30 \pm$ & 0.83 \\
\hline 30. & LBF 90 & $65.91 \pm 0.94$ & $59.95 \pm$ & 0.62 & $33.97 \pm$ & 0.84 \\
\hline 31. & LBF91 & $65.87 \pm 1.06$ & $56.43 \pm$ & 0.69 & $41.19 \pm$ & 0.72 \\
\hline 32. & LBF92 & $62.47 \pm 1.09$ & $54.78 \pm$ & 0.89 & $33.18 \pm$ & 0.81 \\
\hline 33. & LBF93 & $63.87 \pm 1.05$ & $57.59 \pm$ & 0.88 & $31.27 \pm$ & 0.80 \\
\hline 34. & LBF102 & $61.47 \pm 0.87$ & $56.34 \pm$ & 0.88 & $36.25 \pm$ & 0.73 \\
\hline 35. & LBF113 & $64.07 \pm 0.81$ & $56.38 \pm$ & 0.91 & $37.47 \pm$ & 0.77 \\
\hline 36. & LBF119 & $63.56 \pm 0.79$ & $58.49 \pm$ & 0.84 & $38.12 \pm$ & 0.80 \\
\hline 37. & LBF124 & $62.16 \pm 0.86$ & $53.31 \pm$ & 0.83 & $35.37 \pm$ & 0.72 \\
\hline 38. & LBF197 & $71.50 \pm 0.94$ & $65.33 \pm$ & 0.84 & $35.40 \pm$ & 0.60 \\
\hline 39. & LBF198 & $69.40 \pm 0.91$ & $56.59 \pm$ & 0.72 & $33.37 \pm$ & 0.75 \\
\hline
\end{tabular}

Values are means of triplicates in separate runs $n=3 ; \pm$ refers to Standard error of means. 
Table. 2 Survival of Lactobacillus isolates after 3 hours of exposure to simulated gastric juices (Pepsin $3 \mathrm{~g} / \mathrm{L} \mathrm{pH} \mathrm{2.0)} \mathrm{and} \mathrm{simulated} \mathrm{gastric} \mathrm{juices} \mathrm{(Pancreatin} 1.0 \mathrm{~g} / \mathrm{L} \mathrm{pH} \mathrm{8.0)}$

\begin{tabular}{|c|c|c|c|}
\hline S. No. & Isolate No & $\begin{array}{c}\text { Pepsin } 3 \mathrm{~g} / \mathrm{L} \mathrm{pH} \\
2.0\end{array}$ & Pancreatin $1.0 \mathrm{~g} / \mathrm{L}$ pH 8.0 \\
\hline & LGG & $68.13 \pm 0.84$ & $22.19 \pm 0.27$ \\
\hline 1. & LBF 01 & $69.31 \pm 0.78$ & $23.30 \pm 0.26$ \\
\hline 2. & LBF 05 & $61.84 \pm 0.69$ & $18.35 \pm$ \\
\hline 3. & LBF 06 & $69.61 \pm 1.06$ & $5.30 \pm$ \\
\hline 4. & LBF 08 & $66.85 \pm 0.96$ & $16.23 \pm$ \\
\hline 5. & LBF 11 & $69.26 \pm 1.01$ & $15.26 \pm 0.22$ \\
\hline 6. & LBF 13 A & $58.11 \pm 0.66$ & $18.19 \pm$ \\
\hline 7. & LBF 13 B & $52.76 \pm 0.56$ & $10.26 \pm$ \\
\hline 8. & LBF 18 & $59.27 \pm 0.57$ & $9.35 \pm$ \\
\hline 9. & LBF 19 & $58.63 \pm 0.38$ & $8.64 \pm$ \\
\hline 10. & LBF 20 & $61.13 \pm 0.52$ & $21.26 \pm$ \\
\hline 11. & LBF 23 & $65.38 \pm 0.67$ & $12.38 \pm$ \\
\hline 12. & LBF 24 & $64.52 \pm 0.55$ & $11.73 \pm \quad 0.10$ \\
\hline 13. & LBF 26 & $59.62 \pm 0.49$ & $10.99 \pm 0.09$ \\
\hline 14. & LBF35 & $56.59 \pm 0.42$ & $1.59 \pm$ \\
\hline 15 . & LBF53 & $18.97 \pm 0.09$ & $0.70 \pm$ \\
\hline 16. & LBF59 & $61.47 \pm 0.73$ & $11.20 \pm \quad 0.13$ \\
\hline 17. & LBF81 & $52.2 \pm 0.36$ & $12.36 \pm$ \\
\hline 18. & LBF82 & $54.73 \pm 0.42$ & $11.82 \pm \quad 0.09$ \\
\hline 19. & LBF83 & $60.46 \pm 0.41$ & $10.63 \pm 0.07$ \\
\hline 20. & LBF84 & $69.71 \pm \quad 0.45$ & $9.32 \pm$ \\
\hline 21. & LBF85 & $52.17 \pm 0.43$ & $12.63 \pm 0.10$ \\
\hline 22. & LBF87 & $55.52 \pm 0.34$ & $15.14 \pm \quad 0.09$ \\
\hline 23. & LBF89 & $68.12 \pm 0.62$ & $18.67 \pm$ \\
\hline 24. & LBF 90 & $59.29 \pm 0.73$ & $19.83 \pm \quad 0.24$ \\
\hline 25 . & LBF91 & $59.49 \pm 0.35$ & $16.60 \pm \quad 0.10$ \\
\hline 26. & LBF92 & $72.53 \pm 0.50$ & $6.24 \pm$ \\
\hline 27. & LBF93 & $16.21 \pm 0.04$ & $0.30 \pm \quad 0.01$ \\
\hline 28. & LBF102 & $11.42 \pm 0.07$ & $0.47 \pm \quad 0.01$ \\
\hline 29. & LBF113 & $18.17 \pm 0.10$ & $0.79 \pm \quad 0.01$ \\
\hline 30. & LBF119 & $6.71 \pm 0.06$ & $0.91 \pm \quad 0.01$ \\
\hline 31. & LBF124 & $67.46 \pm 0.45$ & $11.66 \pm$ \\
\hline 32. & LBF197 & $10.22 \pm 0.06$ & $9.81 \pm 0.06$ \\
\hline 33. & LBF198 & $73.16 \pm 0.54$ & $13.16 \pm 0.10$ \\
\hline
\end{tabular}

Values are means of triplicates in separate runs $n=3 ; \pm$ refers to Standard error of means. 
Int.J.Curr.Microbiol.App.Sci (2017) 6(3): 1655-1675

Table.3 Cell surface hydrophobicity (\%) of Lactobacillus isolates

\begin{tabular}{lcr}
\hline S. No. & Isolates & \multicolumn{1}{c}{ CSH $(\boldsymbol{\%})$} \\
\hline & LGG & $74.04 \pm 2.24$ \\
1. & LBF 01 & $64.84 \pm 2.39$ \\
2. & LBF 05 & $65.40 \pm 2.15$ \\
3. & LBF 06 & $7.36 \pm 1.99$ \\
4. & LBF 08 & $63.35 \pm 2.05$ \\
5. & LBF 11 & $62.72 \pm 2.09$ \\
6. & LBF 13 A & $56.71 \pm 2.16$ \\
7. & LBF 13 B & $11.00 \pm 0.87$ \\
8. & LBF 18 & $13.82 \pm 0.65$ \\
9. & LBF 19 & $21.65 \pm 1.08$ \\
10. & LBF 20 & $58.90 \pm 2.06$ \\
11. & LBF 23 & $2.39 \pm 0.20$ \\
12. & LBF 24 & $2.99 \pm 0.54$ \\
13. & LBF 26 & $6.03 \pm 0.56$ \\
14. & LBF35 & $7.61 \pm 0.27$ \\
15. & LBF53 & $4.70 \pm 0.80$ \\
16. & LBF81 & $4.56 \pm 0.14$ \\
17. & LBF82 & $7.19 \pm 0.51$ \\
18. & LBF83 & $12.63 \pm 0.78$ \\
19. & LBF84 & $3.39 \pm 0.23$ \\
20. & LBF85 & $2.12 \pm 0.17$ \\
21. & LBF87 & $5.71 \pm 0.15$ \\
22. & LBF89 & $73.48 \pm 2.15$ \\
23. & LBF90 & $71.61 \pm 1.90$ \\
24. & LBF91 & $72.28 \pm 1.69$ \\
25. & LBF92 & $1.71 \pm 0.12$ \\
26. & LBF124 & $2.94 \pm 0.15$ \\
27. & LBF198 & $5.36 \pm 0.10$ \\
\hline
\end{tabular}

Values are means of triplicates in separate runs $n=3 ; \pm$ refers to Standard error of means 
Int.J.Curr.Microbiol.App.Sci (2017) 6(3): 1655-1675

Table.4 Cell auto-aggregation and Co-aggregation ability of selected Lactobacillus isolates

\begin{tabular}{|c|c|c|c|c|c|c|c|}
\hline \multirow{2}{*}{$\begin{array}{l}\text { S. } \\
\text { NO. }\end{array}$} & \multirow[b]{2}{*}{ Isolate } & \multirow{2}{*}{$\begin{array}{c}\text { Auto-aggregation } \\
(\%)\end{array}$} & \multicolumn{4}{|c|}{ Co-aggregation (\%) } & \multirow[b]{2}{*}{$\begin{array}{c}\text { Mean Co- } \\
\text { aggregation }\end{array}$} \\
\hline & & & E. coli & L. monocytogenes & S. abony & S. aureus & \\
\hline & LGG & $50.68 \pm 1.085$ & $48.49 \pm 0.31$ & $72.01 \pm 0.34$ & $37.77 \pm 0.30$ & $48.03 \pm 0.30$ & $51.57 \pm 0.51$ \\
\hline 1. & LBF 01 & $44.66 \pm 1.077$ & $12.09 \pm 0.29$ & $17.85 \pm 0.29$ & $4.30 \pm 0.28$ & $9.59 \pm 0.32$ & $10.96 \pm 0.49$ \\
\hline 2. & LBF 05 & $42.86 \pm 0.910$ & $9.91 \pm 0.29$ & $17.68 \pm 0.27$ & $10.63 \pm 0.28$ & $7.73 \pm 0.26$ & $11.49 \pm 0.43$ \\
\hline 3. & LBF 08 & $41.03 \pm 0.746$ & $26.49 \pm 0.28$ & $30.63 \pm 0.26$ & $24.74 \pm 0.25$ & $21.68 \pm 0.30$ & $11.28 \pm 0.43$ \\
\hline 4. & LBF 11 & $40.50 \pm 0.893$ & $8.34 \pm 0.26$ & $17.25 \pm 0.27$ & $6.43 \pm 0.26$ & $13.11 \pm 0.34$ & $15.77 \pm 0.44$ \\
\hline 5. & LBF 13A & $48.01 \pm 0.893$ & $11.39 \pm 0.24$ & $25.86 \pm 0.22$ & $12.92 \pm 0.27$ & $12.90 \pm 0.28$ & $17.50 \pm 0.43$ \\
\hline 6. & LBF 20 & $43.38 \pm 0.969$ & $23.59 \pm 0.27$ & $31.63 \pm 0.26$ & $14.56 \pm 0.32$ & $0.22 \pm 0.32$ & $50.17 \pm 0.50$ \\
\hline 7. & LBF 89 & $50.67 \pm 1.081$ & $55.74 \pm 0.30$ & $53.96 \pm 0.34$ & $45.10 \pm 0.28$ & $45.90 \pm 0.32$ & $33.56 \pm 0.48$ \\
\hline 8. & LBF 90 & $48.99 \pm 0.972$ & $43.53 \pm 0.33$ & $25.09 \pm 0.33$ & $31.74 \pm 0.27$ & $33.88 \pm 0.32$ & $30.72 \pm 0.46$ \\
\hline \multirow[t]{2}{*}{9.} & LBF 91 & $46.52 \pm 0.902$ & $35.99 \pm 0.35$ & $19.44 \pm 0.39$ & $24.47 \pm 0.32$ & $42.98 \pm 0.34$ & $30.72 \pm 0.46$ \\
\hline & Mean & $45.73 \pm 0.95$ & $27.56 \pm 0.95$ & $21.27 \pm 0.28$ & $31.14 \pm 0.27$ & $23.60 \pm 0.31$ & \\
\hline
\end{tabular}


Table.5 Bile salt hydrolase activity of Lactobacilli cultures

\begin{tabular}{ccccccc}
\hline \multirow{2}{*}{$\begin{array}{l}\text { Lactobacilli } \\
\text { cultures }\end{array}$} & A & B & C & D & E & BSH Activity* \\
\hline LBF 01 & + & + & - & - & - & $6.56 \pm 0.10$ \\
LBF 05 & + & + & - & - & - & $6.17 \pm 0.02$ \\
LBF 08 & + & + & - & - & - & $4.89 \pm 0.08$ \\
LBF 11 & + & + & - & - & - & $4.67 \pm 0.07$ \\
LBF 13 & + & + & - & - & - & $4.93 \pm 0.16$ \\
LBF 20 & + & + & - & - & - & $3.27 \pm 0.17$ \\
LBF 89 & + & + & - & + & + & $2.16 \pm 0.16$ \\
LBF 90 & + & + & - & - & - & $1.89 \pm 0.29$ \\
LBF 91 & + & + & - & + & + & $7.21 \pm 0.10$ \\
LGG & + & + & - & - & + & $5.55 \pm 0.23$ \\
\hline
\end{tabular}

A-Na-taurodeoxycholate hydrate; B-Sodium taurocholate; C-Sodium deoxycholate; D-deoxycholic acid; E-Cholic acid; + Precipitation; - No precipitation; * Values are means of triplicates in separate runs $\mathrm{n}=3 ; \pm$ refers to Standard error of means

Table.6 Results of BLAST search and identification of isolates

\begin{tabular}{llcccll}
\hline S. No. & $\begin{array}{c}\text { Isolate } \\
\text { No }\end{array}$ & $\begin{array}{c}\text { Max } \\
\text { Score }\end{array}$ & T Score & Identity & Name of isolate & Accession No. \\
\hline 1. & LGG & 2643 & 2643 & $98 \%$ & L. rhamnosus & \\
2. & LBF 01 & 2381 & 2381 & $95 \%$ & L. fermentum & KY000526.1 \\
3. & LBF 05 & 2564 & 2564 & $99 \%$ & L. helveticus & KY235775.1 \\
4. & LBF 08 & 2669 & 2669 & $99 \%$ & L. acidophilus & KY235790.1 \\
5. & LBF 11 & 2603 & 2603 & $99 \%$ & L. casei & KY249640.1 \\
6. & LBF 13A & 2741 & 2741 & $99 \%$ & L. fermentum & KY249642.1 \\
7. & LBF 20 & 1531 & 2593 & $100 \%$ & L. reuteri & Awaiting \\
8. & LBF 89 & 2595 & 2595 & $99 \%$ & L. paraplantarum & KY249643.1 \\
9. & LBF 90 & 1548 & 2570 & $100 \%$ & L. fermentum & KY249655.1 \\
10. & LBF 91 & 2599 & 2599 & $99 \%$ & L. planterum & KY249654.1 \\
\hline
\end{tabular}


Fig.1 Amplified PCR products of Lactobacillus isolates showing a PCR product of $250 \mathrm{bp}$

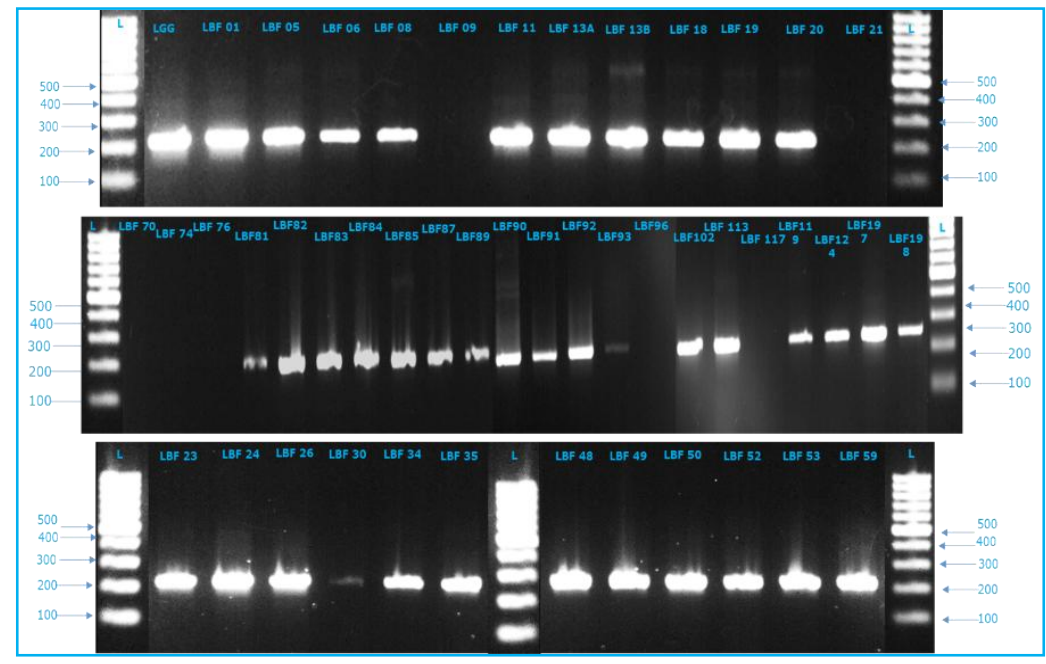

Fig.2 Amplification of the 16S rRNA gene using the bacterial universal primer with expected amplification products comprising sizes of approximately $1500 \mathrm{bp}$

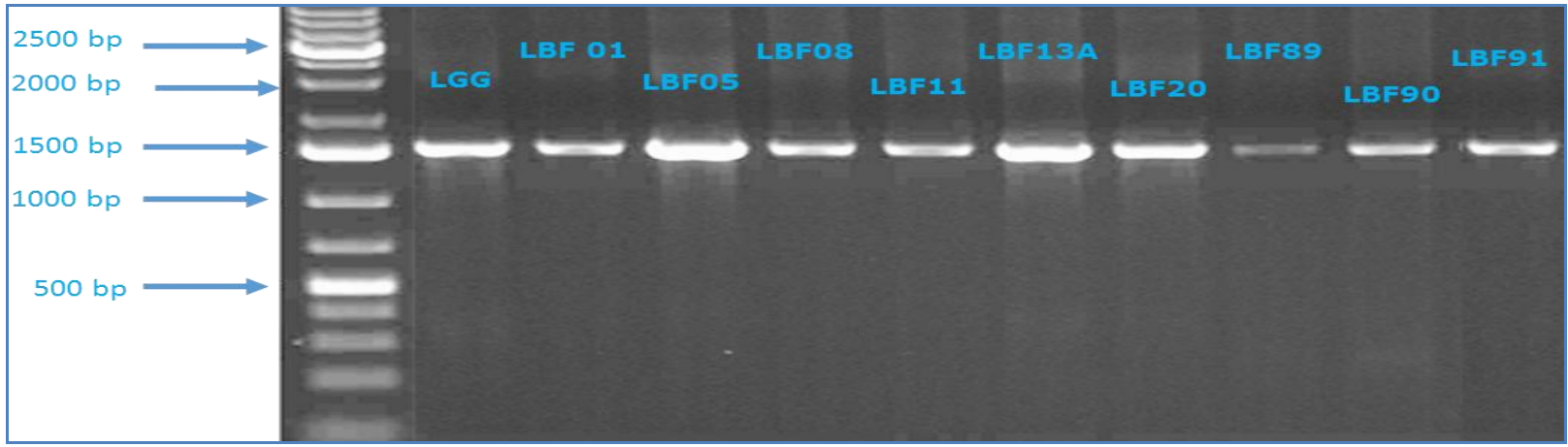

Fig.3 Phylogenetic analysis of isolates

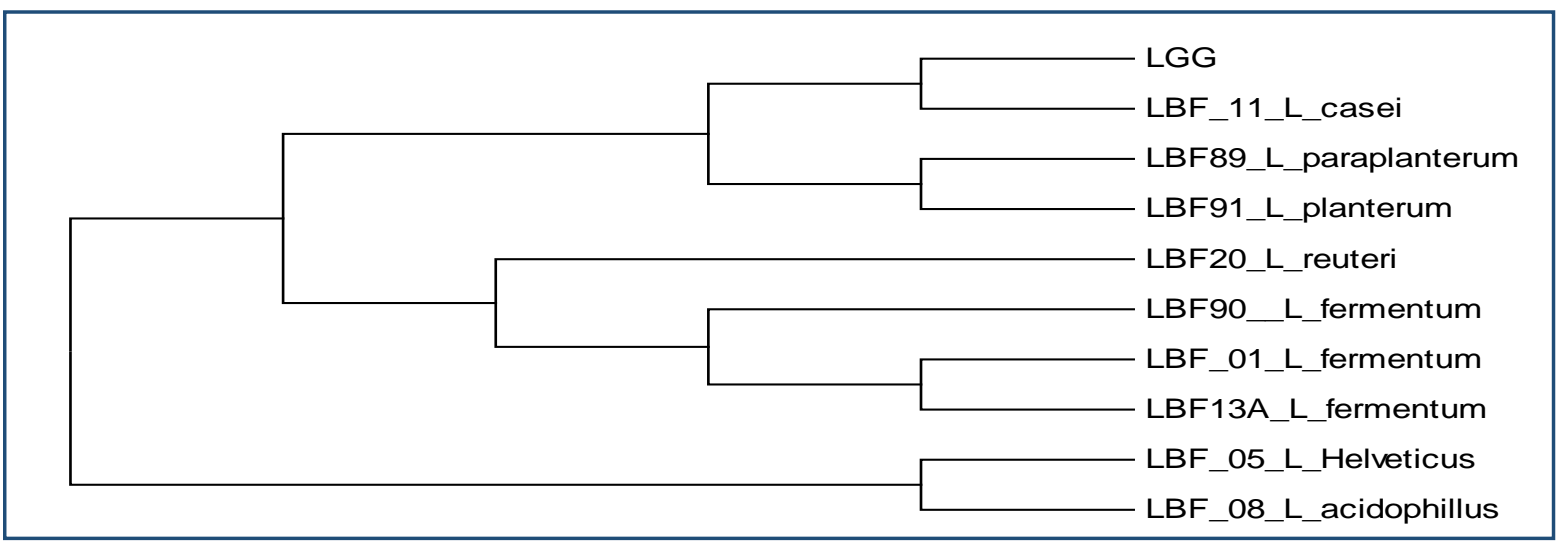


Genus-specific primer with a universal primer, has been tested for its specificity with 23 strains of lactobacilli (Senan, et al., 2008; Kaushik et al., 2009 and Puniya et al., 2008). The studies concluded that all the 39 isolates furnished an amplified product of $250 \mathrm{bp}$ and were characterized as lactobacilli. The same set of primers was also evaluated for identification of Lactobacillus isolates of fecal origin. After comparing the four genus specific primers, LbLMA1/R 16 -1 was found highly specific to Lactobacillus (Senan et al., 2008). Chou and Weimer (1999) opted two fold selection criteria i.e. acid and bile for selection of potentially probiotic isolates. Leyer and Johnson (1993) and Lin et al., (2006) suggested that as and when bile stress takes place after $\mathrm{pH}$ stress, sub-lethally injured microorganisms may have a different and unpredictable conflict to new stress. Davenport (1977) reported that bile concentrations in the intestine range between 0.5 to $2.0 \%$ during first hour of digestion while the levels may decrease during the second hour. Kingwatee et al., (2014) concluded that, no viable cells could be observed after $30 \mathrm{~min}$ of incubation on different bile salt concentrations $(0.3 \%, 0.5 \%$ and $1 \%$ ) while observed with Lactobacillus casei 01 indicated that the strain is very sensitive to the concentration used. On other hand Buruleanu (2012) concluded that the mortality of the lactic acid bacteria was by 0.9 $\log$ cells after $5 \mathrm{~h}$ of incubation at initial concentration $(0.1 \%)$ of bile while a mortality rate of $1.4 \log$ cells was observed with the $0.3 \%$ of bile concentration.

In present investigation, selected Lactobacillus isolates were able to withstand extreme gastric as well as intestinal conditions efficiently, therefore can be recommended as potential probiotic candidates for further use as direct dietary supplements as well as in fermented food preparations to improve the gut health. Zhang et al., (2013) reported $8.76 \%$ survival in presence of trypsinase concentration at 7.0 g/L. Vamanu, et al., (2011) reported that the viability of Lactobacillus rhamnosus IL4.2 strain under $0.5 \% \mathrm{NaCl}$. The influence of pepsin $(3 \mathrm{~g} / \mathrm{L}$; with variable $\mathrm{pH}(1.5,2,2.5 \&$ $3)$ as well as of pancreatin $(1 \mathrm{~g} / \mathrm{L})$ in the presence of bile salts $(1.5,2.0,3.0 \& 5.0$ $\mathrm{mg} / \mathrm{mL}$ ) were studied. The survival of $L$. plantarum WCFS1 in the presence of pancreatic enzymes up to $4 \mathrm{~h}$ indicated its potential to survive under the harsh environment in the small intestines in comparison to L. casei BD II (Quinto, et al., 2003).

Autoaggregation and hydrophobicity has been applied as a measurement of the ability of bacteria to adhere to cell monolayers (Bautista-Gallego et al., 2013). In same direction, a correlation between hydrophobicity and adhesion ability has been observed (Ehrmann et al., 2002), while some reports has concluded that hydrophobicity values do not correlate with adhesion properties (Ramos et al., 2013). It has been suggested that bacterial cells with a high hydrophobicity usually present strong interactions with mucosal cells. Cell surface hydrophobicity of the cell is directly proportional to the adhesion to gut epithelial cells. This is because of greater attractive forces and smaller (more negative) electro kinetic potentials of cells and solids (Rijnaarts et al., 1993).

L. fermentum JMC 7776 sourced from infant fecal samples revealed $59.58 \%$ hydrophobicity in toluene and $44.26 \%$ in xylene, while L. fermentum 39-183 isolated from traditional fermented foods showed $25.01 \%$ hydrophobicity with toluene, and $22.43 \%$ in xylene (Ramos, et al., 2013). Puniya et al., (2012) also observed the highest hydrophobicity for L. casei ranging from $36 \%$ to $56 \%$. Differences in the $\mathrm{CSH}$ could 
attributed to variable expression of cell surface proteins among different strains of a species as well as to environmental conditions that could affect the expression of cell surface proteins (Kaushik et al., 2009). Hence, based on cell surface hydrophobicity, only 9 isolates (LBF 01, LBF 05, LBF 08, LBF 11, LBF 13 A, LBF 20, LBF89, LBF90, LBF91) were selected for further characterization.Cell adhesion, a multistep process, involves contact of the bacterial cell membrane and intermingling surfaces while aggregation may take place between cells of the same strain (auto-aggregation) or between different species and strains (co-aggregation). Aggregation has been considered as an important mechanism for genetic exchange, adhesion, and colonization in the host environments, as well as Immuno modulation of colonic mucosa (Cesena et al., 2001, Voltan et al., 2007).

Savedboworn et al., (2014) concluded that most of the LAB exhibited a strong autoaggregation after $5 \mathrm{~h}$ of incubation like KMUTNB 5-8 strain showed highest autoaggregation ability of $96.09 \%$. On another hand some isolates (KMUTNB 5-27) could not show any auto-aggregation. Probiotic and pathogenic bacteria have been reported to form joint aggregate and the process is known as co-aggregation (Surono, 2004) resulting in effectively inhibiting and killing them by secreting antimicrobial compounds that act directly on pathogens (Bao et al., 2010). Li et al., (2015) studied the co-aggregation ability of 18 lactic acid bacteria isolated from traditional fermented foods and found all the isolates to have co-aggregation ability with Salmonella sp. ranging from $5.15 \%$ to $29.54 \%$. Co-aggregation of L. acidophilus M92 with two other potential probiotic strains (L. plantarum L4, E. faecium L3) and two enteropathogens ( $S$. abonymurium and $E$. coli) was examined wherein $L$. acidophilus M92 exhibited $4.36 \%$ co-aggregation with $L$. plantarum L4, $19.46 \%$ with E. faecium L3, $15.11 \%$ with $E$. coli 3014 and $15.70 \%$ with $S$. abonymurium (Kos et al., 2003).

The efficient co-aggregation ability of probiotic bacteria against gram positive bacteria could be attributed to the similar cell wall morphology of LAB and gram positive pathogens and their hydrophobic nature making it easier to bond altogether (Arief et al., 2015). Moreover, lactic acid bacteria strains could control the microenviroment around the pathogens and increase the concentration of excreted antimicrobial substances in the process of co-aggregation (Li et al., 2015) which constitute an important host defense mechanism against infection in the gastrointestinal tract (Reid et al., 1988).

Free bile acids formed by the deconjugation of conjugated bile salts are less soluble and are less likely to be reabsorbed by the intestinal lumen compared to their conjugated equivalent, and are lost from the human body through feces (Center, 1993). Ahn et al., (2003) and Begley et al., (2006), described precipitation of bile salt by BSH activity of probiotic strains. Bile salt hydrolase activity against sodium tauroglycocholate but low intensity of precipitation was noticed as compared to sodium taurodeoxycholate. Probiotics such as $L$. acidophilus have been reported to possess bile salt hydrolase (BSH) or cholylglycine hydrolase (the enzyme that catalyzes the hydrolysis of glycine- and taurine-conjugated bile salts into amino acid residues and free bile salts). BSH has been reported to be present in several bacterial species of the gastrointestinal tract, such as Lactobacillus sp.,B. longum, C. perfringens and $B$. fragilis ssp. fragilis(Corzo \& Gilliland, 1999). Human intestinal $\mathrm{pH}$ of 6.5 and a glycocholate to taurocholate ratio of $2: 3$ were found glycine conjugated bile salt to be more efficiently deconjugated by strains of $L$. acidophilus from both human and porcine 
origins than taurine conjugated bile salt(Corzo \& Gilliland,1999). L. buchneri JCM 1069 and Lactobacillus kefir BCCM 9480 expressed substrate specific BSH based on the structure of the steroid moiety of the bile salt conjugate (De Smet et al., 1995; Moser \& Savage, 2001). Brashears et al., (1998) postulated if the deconjugation mechanism is important in decreasing serum cholesterol then bacterial strains that prefer to deconjugate sodium glycocholate, may have more potential to lower serum cholesterol concentrations and hence reducing the risk of heart problems. Accordingly, Liong and Shah (2005) concluded that L. acidophilus ATCC 33200, 4357, 4962 and L. casei ASCC 1521 possess high deconjugation activity towards sodium glycocholate and sodium taurocholate and hence may exert better in vivo deconjugation properties. Similarly, it can be concluded that the isolates, especially LBF 08, LBF 89, LBF90 and LBF 91 may be explored for controlling the serum cholesterol after in-vivo experiments.

The nucleotide sequence of $16 \mathrm{~S}$ ribosomal DNA (rDNA) not only provides accurate and specific identification of unknown isolates but also helps to study the diversity of the microbiological population (Drancourt et al., 2000; Greetham et al., 2002; Heilig et al., 2002).

Biodiversity of Lactobacillus genus by S-GLab-0677- a-A-17 in combination with primer Bact-0011f on bacterial DNA isolated from fecal and other intestinal samples resulting in a 700 bp PCR products has been studied by Heilig et al., (2002). Sakamoto et al., (2011) reported that Lactobacillus species, including $L$. namurensis and $L$. acetotolerans, predominate the long aged nukadoko, a traditional Japanese fermented rice bran bed used for pickling vegetables while investigated with molecular tools. Moreover, Zubaidah et al., (2012) isolated L. plantarum from fermented rice bran for its synbiotic effect and based on phylogenetic analysis concluded that most strains isolated from fermented rice bran products are highly similar to $L$. johnsonii. Adeyemo and Onilude, (2014) isolated $20 \mathrm{~L}$. plantarum from spontaneously fermented cereals and identified using classical methods as well as molecular methods by amplification of $16 \mathrm{~S}$ rDNA genes. The author concluded that $15 \%$ of isolates were misidentified while used conventional approach. $16 \mathrm{~S}$ r RNA gene sequencing is one of the most reliable molecular tools for identification of bacterial isolates because it's one of the highly conserved region of an organism's genome and hence has been targeted for molecular identification of isolates.

In conclusion LAB play an important role in the majority of food fermentations, and a wide variety of strains are routinely employed as starter cultures in the manufacture of dairy, meat, vegetables and bakery products. The preparation of indigenous fermented food generally depends on a spontaneous or chance inoculation by naturally occurring LAB and application of starter cultures is still at very early stages. One of the major influences of these microorganisms is the extended shelf life of the fermented product by comparison to that of the raw substrate. Among the bacteria producing antimicrobials, LAB has fascinated investigators very much as they enjoy GRAS status. In the present study, 46 acid tolerant Lactobacillus isolates, sequestered from different sources were taken into consideration wherein only 9 isolates were selected on the basis of their high probiotic attributes. Among these 9 isolates, two isolates LBF 89 and LBF 91 had the highest bile tolerance and further in-vitro assessment reveled that it also showed high tolerance in gastrointestinal tract. Moreover, these two isolates have significant level of cell surface hydrophobicity, cell auto- 
aggregation and preferable co-aggregation properties.

These isolates also demonstrated high level of bile salt hydrolase activity which is very important factor for hypocholesterolemic effect. Both the isolates were identified as Lactobacillus planterum but distantly placed in the phylogenetic tree. Accordingly, the isolates could be potentially used in functional food and health products especially where cholesterol reduction infood is the main target. Further in vivo study is required to establish the hypocholesterolemic effect and its mechanism(s) involved in the reduction of cholesterol by such promising isolates.

\section{References}

Adeyemo, S.M. and Onilude, A.A. 2014. Molecular identification of Lactobacillus plantarum isolated from fermenting cereals. Int. J. Biotechnol. Mol. Biol. Res., 56: 59-67.

Ahn, Y.T., Kim, G.B. and Lim, Y.S. 2003. Deconjugation of bile salts by Lactobacillus acidophilus isolates. Int. Dairy. J. 13: 303-311.

Altschul, S.F., Madden, T.L., Schaffer, A.A., Zhang, J., Zhang, Z., Miller, W. and Lipman, D.J., 1997. Gapped BLAST and PSI-BLAST: a new generation of protein database search programs. $\mathrm{Nucl}$. Acids Res., 25: 3389-3402.

Angmo, K., Kumari, A., Bhalla, T.C., 2016. Probiotic characterization of lactic acid bacteria isolated from fermented foods and beverage of Ladakh. LWT-Food. Sci. Technol., 66: 428-435.

Arief I I, Jenie B S L, Astawan M, Fujiyama $\mathrm{K}$ and Witarto A B. 2015. Identification and probiotic characteristics of lactic acid bacteria isolated from Indonasian local beef. Asian J. Anim. Sci., 9(1): 25-36.
Bao, Y., Zhang, Y., Zhang, Y., Liu, Y., Wang, S., Dong, X., Wang, Y., Zhang, H. 2010. Screening of potential probiotic properties of Lactobacillus fermentum isolated from traditional dairy products. Food Control, 21: 695701.

Bautista-Gallego, J., Arroyo-L'opez, F.N., Rantsiou, K., Jimenez-Diaz, R., Garrido-Fernandez, A., and Cocolin, L. 2013. Screening of lactic acid bacteria isolated from fermented table olives with probiotic potential. Food. Res. Int., 50) 1: 135-142.

Begley, M., Hill, C. and Gahan, C.G.M. 2006. Bile salt hydrolase activity in probiotics. Appl. Environ. Microbiol., 72(3): 17291738.

Brashears, M.M., Gilliland, S.E. and Buck, L.M. 1998: Bile salt deconjugation and cholesterol removal from media by Lactobacillus casei. J. Dairy Sci., 81: 2103-2110.

Buruleanu, J.L. 2012. Acid and bile tolerance of probiotic bacteria used for lactic acid fermentation of vegetable. J. Sci. Arts., 118: 57-62.

Center, S. A. Ed. 1999. Serum bile acid in companion animal medicine. In Micheal, S. L. Gastroenterology: The 1990s pp. 625-657. Philadelphia: Saunders.

Cesena, C., L. Morelli, M. Alander, T. Siljander, E. Tuomola, S. Salminen, T. Mattila-Sandholm, T. VilpponenSalmela, and A. von Wright. 2001. Lactobacillus crispatus and its nonaggregating mutant in human colonization trials. J. Dairy Sci., 84: 1001-1010.

Chou, L.S. and Weimer, B. 1999. Isolation and characterization of acid- and biletolerant isolates from strains of Lactobacillus acidophilus. J. Dairy Sci., 82: 23-31.

Collins, M.D. and Gibson, G.R., 1999. 
Probiotics, prebiotics and synbiotics: approaches for modulating the microbial ecology of the gut. Am. J. Clin. Nutr., 69: 1052S-1057S.

Corzo, G., and Gilliland, S. E. 1999. Bile salt hydrolase activity of three strains of Lactobacillus acidophilus. J. Dairy Sci., 82: 472-480.

Davenpot, H.W., Ed. 1997). Physiology of the digestive tract. $4^{\text {th }}$ Ed. Year Book Medical Publishers Incorporated, Chicago, IL. pp. 232

De, Smet. I., Van. Hoorde. L., Vande. Woestyne. M., Cristianes. H. and Verstraete. W. 1995. Significance of bile salt hydrolytic activities of Lactobacilli. J. Appl. Bacteriol., 79:292-30.

Del Re, B., Sgorbati, B., Miglioli, M. and Palenzona, D. 2000. Adhesion autoaggregation and hydrophobicity of 13 strains of Bifidobacterium longum. Lett. Microbiol., 31: 438-442.

Drancourt, M., Bollet, C., Carlioz, A., Martelin, R., Grayral, J.P. and Raoult, D. 2000. 16S Ribosomal DNA sequence analysis of a large collection of environmental and clinical unidentifiable bacterial isolates. J. Clin. Microbiol., 38: 3623-3630.

Dubernet, S., Desmasures, N. and Guéguen, M. 2002. A PCR-based method for identification of lactobacilli at the genus level, FEMS Microbiol. Lett., 214: 271275.

Dunne, C., O’Mahony, L., Murphy, L., Thornton, G., Morrissey, D., O’Halloran, S., Feeney, M., Flynn, S., Fitzgerald, G., Daly, C., Kiely, B., O'Sullivan, G.C., Shanahan, F. and Collins, J.K. 2001. In vitro selection criteria for probiotic bacteria of human origin: correlation with in vivo findings. M. J. Clin. Nutr., 73(2): 386-392

Ehrmann, M.A., Kurzak, P., Bauer, J., and Vogel, R.F. 2002. Characterization of lactobacilli towards their use as probiotic adjuncts in poultry. J. Appl. Microbiol., 92(5): 966-975.

Fernandez, M.F., Boris, S. and Barbes, C. 2003. Probiotic properties of human lactobacilli strains to be used in the gastrointestinal tract. J. Appl. Microbiol., 94: 449- 455.

Gilliland, S.E., Staley, T.E., Bush, L.J. 1984. Importance of bile tolerance of Lactobacillus acidophilus used as dietary adjuct. J. Dairy. Sci., 67:30453055

Greetham, H.L., Giffard, C., Hutson, R.A., Collins, M.D. and Gibson, G. R., 2002. Bacteriology of the Labrador dog gut: a cultural and genotypic approach. $J$. Appl. Microbiol., 93: 640-646.

Gupta, A. 2015. Characterization of potentially active new probiotic strains isolated from different sources and to study their prospects as nutraceutical agents. Doctoral Thesis, Dr. Yashwant Singh Parmar University of Horticulture and Forestry, Solan, India.

Handley, P.S., Harty, D.W.S., Wyatt, J.E., Brown, C.R., Doran, J.P., Gibbs, A.C.C. 1987. A comparison of the adhesion, coaggregation and cell-surface hydrophobicity properties of fibrillar and fimbriate strains of Streptococcus salivarius.J. Gen. Microbiol., 133: 3207-3217.

Heilig, H.G., Zoetendal, E.G., Vaughan, E.E., Marteau, P., Akkermans, A.D., de Vos, W.M. 2002. Molecular diversity of Lactobacillus spp. and other lactic acid bacteria in the human intestine as determined by specific amplification of $16 \mathrm{~S}$ ribosomal DNA. Appl. Environ. Microbiol., 68: 114-123.

Kaushik, J.K., Kumar, A., Duary, R.K., Mohanty, A.K. and Grover, S. 2009. Functional and Probiotic Attributes of an Indigenous Isolate of Lactobacillus plantarum. PLoS ONE, 412: 8099. 
Kingwatee, N., Apichartsrangkoon, A., Chaikham, P., Pankasemsuk, T. and Changrue, V. 2014. Survivability and metabolic activity of Lactobacillus casei 01 incorporating lychee juice plus inulin under simulated gastrointestinal environment. Int. Food. Res. J., 211: 83-89.

Kos, B., Suskovic, J., Goreta, J. and Matosic, S. 2000. Effect of Protectors on the Viability of Lactobacillus acidophilus M92 in Simulated Gastrointestinal Conditions. Food. Technol. Biotechnolo., 382: 121-127.

Kos, B., Suskovic, J., Vukovic, S., Simpraga, M., Frece, J. and Matosic, S. 2003. Adhesion and aggregation ability of probiotic strain Lactobacillus acidophilus M92. J. Appl. Microbiol., 94: 981-987.

Kumar M., Ghosh M. and Ganguli A. 2012. Mitogenic response and probiotic characteristics of lactic acid bacteria isolated from indigenously pickled vegetables and fermented beverages. World J. Microbiol. Biotechnol., 28: 703-711.

Lee, Y.K. and Salminen, S. 1995. The coming of age of probiotics. Trends. Food. Sci. Technol., 6: 241-245.

Leyer, G.L. and Johnson, E.A. 1993. Acid adaptation induces cross-protection against environmental stresses in Salmonella abonymurium. Appl. Environ. Microbiol., 59: 1842.

Li, Q., Liu, X., Dong, M., Zhou, J. and Wang, Y. 2015. Aggregation and adhesion abilities of 18 lactic acid bacteria strains isolated from traditional fermented food. Int. J. Agri. Policy Res. 3(2): 8492.

Lin, W.H., Hwang, C.F., Chen, L.W. and Tsen, H.Y. 2006. Viable counts, characteristic evaluation for commercial lactic acid bacteria products. Food Microbiol., 23: 74-81.
Liong, M.T. and Shah, N.P. 2005 Bile salt deconjugation ability, bile salt hydrolase activity and cholesterol coprecipitation ability of lactobacilli strains. Int. Dairy J., 15: 391-398.

Miremadi, F., Ayyash, M., Sherkat, F., Stojanovska, L., 2014. Cholesterol reduction mechanisms and fatty acid composition of cellular membranes of probiotic Lactobacilli and Bifidobacteria. J. Funct. Foods., 9: 295-305.

Mojgani, N., Fatimah, H.F. and Vaseji, N. 2015. Characterization of indigenous Lactobacillus strains for probiotic properties. Jundishapur J. Microbiol., 8(2):1-2.

Moser, S.A. and Savage, D. 2001. Bile salt hydrolase activity and resistance to toxicity of conjugated bile salts are unrelated properties in lactobacilli. Appl Environ Microbiol 678: 3476-3480.

Niguez-Palomares, C.I., Perez-Morales, R. and Acedo-Felix, E. 2008. Evaluation of probiotic properties in Lactobacillusisolated from small intestine of piglets. Revista Latinoamericana de Microbiologia., 493-4: 46-54.

Park, Y.H., Jong, G.K., Young, W.S., Sae, H.K. and Kwang, Y.W. 2007. Effect of dietary inclusion ofLactobacillus acidophilus ATCC 43121 on cholesterol metabolism in rats. J. Microbiol. Biotechnol., 17: 655-662.

Pennacchia, C., D. Ercolini, G. Blaiotta, O. Pepe, G. Mauriello and F. Villani. 2004. Selection of Lactobacillus strains from fermented sausages for their potential use as probiotics. Meat Sci., 67: 309317.

Prasad, J., Gill, H., Smart, J. and Gopal, P.K. 1998. Selection and characterization of Lactobacillus and Bifidobacterium strains for use as probiotics. Int. Dairy J., 8: 993-1002. 
Puniya, A.K., Chaitanya, S., Tyagi, A. K., De, S and Singh, K. 2008. Conjugated linoleic acid producing potential of lactobacilli isolated from the rumen of cattle. J. Ind. Microbiol. Biotechnol., 35: $1223-122$

Puniya, M., Sangu, K.P.S, Bharadwaj, A, Gupta, D., Kumar, S., Dhewa, T. and Pant, S. 2012. Probiotic and functional attributes of Lactobacillus spp. isolated from human faeces. J. Res. Antimicrobiol., 1: 032-042.

Quinto, E.A., Sahagun, J., Idurot, H., Medina, S. and Sy, G. 2003. Lactobacillus isolate USTCMS 1071: A potential swine probiotic for a safer and cleaner environment. Acta Manilana., 51: 4956.

Ramos, C.L., Thorsen, L., Schwan, R.F. and Jespersen, L. 2013. Strain specific probiotics properties of Lactobacillus fermentum, Lactobacillus plantarum and Lactobacillus brevis isolates from Brazilian food products. Food Microbiol., 36(1): 22-29.

Reid. G., McGroarty, J. A., Angotti, R. and Cook, R. L. 1988. Lactobacillus inhibitor production against Escherichia coli and coaggregation ability with uropathogens. Can. J. Microbiol., 34: 344-351.

Rijnaarts, H.H.M, Norde, W., Bouwer, E.J., Lyklema, J. and Zehnder, A.J.B. 1993. Bacterial adhesion under static and dynamic conditions. Appl. Environ. Microbiol., 59: 3255-3265

Rodas, A. M., Ferrer, S. and Pardo, I. 2003. 16S-ARDRA, a tool for identification of lactic acid bacteria isolated from grape must and wine. Syst. Appl. Microbiol., 26: 412-422.

Rosenberg, M., Gutnick, D. and Rosenberg, E. 1980. Adherence of bacteria to hydrocarbons: A simple method for measuring cell hydrophobicity. FEMS Microbiol. Lett., 9: 29-33.
Salminen, S., von Wright, A., Morelli, L., Marteau, P., Brassart, D., de Vos, W.M., Fonde'n, R., Saxelin, M., Collins, K., Mogensen, G., Birkeland, S.-E. and Mattila-Sandholm, T. 1998 b. Demonstration of safety of probiotics - A review. Int J Food Microbiol., 44: 93-106.

Sanders, M.E., in't Veld, J.H., 1999. Bringing a probiotic-containing functional food to the market: microbiological, product, regulatory and labeling issues. Antonie Van Leeuwenhoek 76:293-315.

Savedboworn, W., Riansa-ngawong, W., Sinlapacharoen, W., Pajakang, S. and Patcharajarukit, B. 2014. Assessment of probiotic properties in lactic acid bacteria isolated from fermented vegetables. Int. J. App. Sci. Tech., 74: 53-65.

Schillinger, U., Guigas, C. and Holzapfel, W.H. 2005. In vitro adherence and other properties of lactobacilli used in probiotic yoghurt like products. Int. Dairy. J., 15:1289-1297.

Senan, S., Grover, S. and Batish, V.K. 2008. Comparison of specificity of different primer pairs for the development of multiplex PCR assays for rapid identification of dairy Lactobacilli. Int. J. Sci. Technol., 32: 123- 137.

Surono, I. S. 2004. Probiotic, fermented milk and healthy. YAPPMI, Jakarta, Inonasia, pp. 20-40.

Tambekar, D.H. and Bhutada, S.A. 2010. Studies on antimicrobial activity and characteristics of bacteriocins produced by Lactobacillus strains isolated from milk of domestic animals. The Internet. J. Microbiol., 8:1-6.

Tamura, K., Stecher, G., Peterson, D., Filipski, A. and Kumar, S. 2013. MEGA6: Molecular Evolutionary Genetics Analysis version 6.0. Mol. Biol. Evol., 30: 2725-9.

Tuomola, E., Crittenden R., Playne M., 
Isolauri E., and Salminen S. 2001. Quality assurance criteria for probiotic bacteria. American J. Clin. Nutrition., 732: 393S-398S.

Vamanu, E., Vamanu, A., Pelinescu, D., Nita, S. and Rusu, N. 2011. The Viability of the Lactobacillus Rhamnosus IL4.2 Strain in Simulated Gastrointestinal Conditions Animal Science and Biotechnologies., 441: 459-464.

Vijaya, K.B., Vijayendra, S.V.N. and Reddy, O.V.S., 2015. Trends in dairy and nondairy probiotic products-A review. $J$. Food Sci. Technol., 52: 6112-6124.

Voltan, S., Castagliuolo, I., Elli, M., Longo, S., Brun, P., D’Inca, R., Porzionato, A., Macchi, V., Palu, G., Sturniolo, G. C., Morelli, L. and Martines. D. 2007. Aggregating phenotype in Lactobacillus crispatus determines intestinal colonization and TLR2 and TLR4 modulation in murine colonic mucosa. Clin. Vaccine Immunol., 14:1138-1148.

Vries, M.C., Vaughan, E.E., Kleerebezem, M. and de Vos, W.M., 2006. Lactobacillus plantarum survival, functional and potential probiotic properties in the human intestinal tract. Int. Dairy J. 16:1018-1028.
Xie, Y., Zhang, H., Liu, H., Xiong, L., Gao, X., Jia, H., Lian, Z., Tong, N. and Han, T. 2015. Hypocholesterolemic effects of Kluyveromyces marxianus M3 isolated from Tibetan mushrooms on diet induced hypercholesterolemia in rat. Brazil. J. Microbiol., 46:389-395.

Yujiang Xiang, Hyun-Joon Chung, Joo H. Kim, Rajankumar Bhatt, Salam Rahmatalla, Jingzhou Yang, Timothy Marler, Jasbir S. Arora and Karim Abdel-Malek. 2010. Predictive dynamics: an optimization-based novel approach for human motion simulation. Struct. Multidisc. Optim. 41(3): 465479.

Zhang, W., Liu, M. and Dai, X. 2013. Biological characteristics and probiotic effect of Leuconostoc lactis strain isolated from the intestine of black porgy fish. Braz. J. Microbiol., 443: 685-691

Zubaidah, E., Nurcholis, M., Wulan, S.N. and Kusuma, A. 2012. Comparative Study on Synbiotic Effect of Fermented Rice Bran by Probiotic Lactic Acid Bacteria Lactobacillus casei and Newly Isolated Lactobacillus plantarum B2 in Wistar Rats," APCBEE Procedia, 2: 170-177.

\section{How to cite this article:}

Pradip Kumar Sharma, Pradeep Kumar Sharma and Naresh Kumar, Suman and Niti Dhingra. 2017. Identification and Characterization of Bile Salt Hydrolyzing Lactobacillus Isolates. Int.J.Curr.Microbiol.App.Sci. 6(3): 1655-1675. doi: https://doi.org/10.20546/ijcmas.2017.603.192 